\title{
Teneurs en hormones dans les tissus des organes aériens de Faidherbia albida (Del) A. Chevalier en saison pluvieuse
}

\author{
Sékeyoba Léopold NANEMA*, Rasmata NANA, Badoua BADIEL, Bibata KONATE, \\ Memti Mberdoum NGUINAMBAYE et Zoumbiéssé TAMINI
}

\author{
Laboratoire Biosciences, Equipe d'Ecophysiologie Végétale, Université de Ouagadougou, 03 BP \\ 7021 Ouagadougou 03, Burkina Faso. \\ *Auteur correspondant, E-mail : leonanema@yahoo.fr; Tel : +226 70273422/+226 78620904
}

\section{RESUME}

La présente étude est axée sur des observations, des prélèvements d'organes sur des sujets sains, l'extraction des hormones et leur dosage spectrophotométrique. Les observations phénologiques de cinq pieds, de juin à octobre, ont permis de noter une défeuillaison de juin à juillet et une feuillaison à partir de fin septembre. Les analyses effectuées sur les feuilles, les écorces et les rameaux révèlent des taux variables de phytohormones. Ainsi, en fonction de l'organe et de la période, la teneur en auxine AIA3 a varié de 0,3 à 1,5 $\mathrm{mg} / \mathrm{kg}$ de matière végétale fraîche, celle en gibbérelline $\mathrm{GA} 3$, de 1,5 à $3 \mathrm{mg} / \mathrm{kg}$ et celle en kinétine de 1,4 à 3 $\mathrm{mg} / \mathrm{kg}$. Cette variation est croissante pour l'AIA3 et la kinétine et décroissante pour la gibbérelline au niveau des écorces. Une tendance similaire est observée au niveau des rameaux. Cependant, dans les feuilles, les trois hormones ont évolué à la hausse à partir de fin juillet. Il existe une corrélation entre ces différentes hormones. La production de l'AIA3 influence négativement sur celle de la gibbérelline. Aucun effet significatif de l'AIA3 et de GA3 sur la kinétine n'est noté. L'objectif est de contribuer à une meilleure connaissance de la physiologie de l'espèce.

() 2015 International Formulae Group. All rights reserved.

Mots clés : Hormones végétales, organes aériens, phénologie foliaire.

\section{INTRODUCTION}

Faidherbia albida (syn Acacia albida) est une espèce originaire d'Afrique et du Moyen-Orient. C'est une légumineuse arborée de la famille des Fabacées bien connue de la plupart des acteurs du développement. Son impact sur la fertilité du sol et sur l'alimentation du bétail a été bien étudié par Khao et al. (2001) puis, par Gnankambary (2007). Selon Harivel et al. (2006), sa multiplication peut se faire également par drageonnage. Dans certaines régions, en conditions naturelles, la propagation par graines demeure la règle. Des essais réalisés au Burkina Faso ont montré que la multiplication végétative par bouturage de Faidherbia albida est possible avec un taux de réussite d'environ $50 \%$. Compte tenu de son potentiel fertilisant à long terme pour les sols et de son apport alimentaire pour le bétail, ainsi que son adaptation aux conditions pédoclimatiques difficiles, son usage a été sérieusement envisagé comme une alternative pour promouvoir un développement agricole durable. Ainsi, la promotion des ressources naturelles constituent une des stratégies pour 
lever les contraintes liées à la productivité des cultures. En raison de cette importance et des perspectives de domestication et d'amélioration génétique qu'il offre, Faidherbia albida a été depuis 1974, classée comme essence prioritaire par le groupe d'experts des ressources génétiques forestières (FAO, 2000). Malheureusement, Faidherbia albida, comme d'autres espèces arborescentes des zones arides et semi-arides, est menacée de disparition par la destruction des écosystèmes naturels. En dépit de l'intérêt de Faidherbia albida pour l'agropastoralisme, les parcs de l'espèce sont depuis plus de 30 ans, en déclin croissant et sa régénération naturelle est pratiquement nulle.

Face aux menaces qui pèsent sur le patrimoine génétique et les perspectives d'amélioration qu'offre Faidherbia albida, il apparaît nécessaire de prendre des mesures pour conserver cette essence précieuse pourvoyeuse de biens et services pour les zones rurales.

Malgré l'apparente profusion des travaux sur Faidherbia albida, sa physiologie reste mal connue et bien de lacunes restent à combler, notamment en ce qui concerne son rythme phénologique particulier. En effet, Faidherbia albida perd ses feuilles en saison pluvieuse contrairement à la plupart des plantes de savane où cette saison correspond à la période d'activité biologique. Ses feuilles réapparaissent dès la fin de la saison pluvieuse (Ouédraogo, 2004).Il n'existe pas à ce jour une explication satisfaisante sur la phénologie particulière de Faidherbia albida et cela reste un défi à relever pour toute la communauté scientifique. Les teneurs en hormones de la plante n'influencent-elles pas sa feuillaison/défeuillaison? A terme, une meilleure compréhension de tous les mécanismes physiologiques permettra de mieux valoriser cette espèce.

C'est dans le but d'apporter des informations complémentaires aux investigations déjà menées sur l'espèce que cette étude a été initiée. Il s'agit particulièrement de contribuer à une meilleure connaissance de la physiologie de l'espèce Faidherbia albida.

De façon spécifique, il s'agit de déterminer les teneurs en phytohormones de Faidherbia albida au niveau des feuilles, de l'écorce et des rameaux, notamment la GA3 du groupe des gibbérellines, la kinétine du groupe des cytokinines et l'acide indolyl-3 acétique (AIA3) qui est une auxine au cours d'une saison pluvieuse.

\section{MATERIEL ET METHODES \\ Site de l'étude}

Le site de l'étude est le village de Gonsé situé entre la longitude ouest $1^{\circ} 19^{\prime}$ et la latitude $12^{\circ} 2^{\prime}$ nord à $20 \mathrm{~km}$ à l'Est de Ouagadougou, la capitale du Burkina Faso (Figure 1). Les critères de choix du site de l'étude ont porté sur la disponibilité des pieds de Faidherbia albida, de leur représentativité et de l'accessibilité du site en saison pluvieuse. La zone d'étude a un relief relativement plat, $300 \mathrm{~m}$ d'altitude et repose sur un socle granitique précambrien avec des sols ferrugineux tropicaux lessivés à tâches et concrétions ou à carapace et cuirasse (Bationo et al. 2001). Le climat de la zone est de type soudano-sahélien avec une saison pluvieuse qui dure quatre (4) mois de juin à septembre et une saison sèche d'octobre à mai. Les précipitations sont irrégulières avec des moyennes annuelles qui varient entre 700 et $800 \mathrm{~mm}$ et une température moyenne annuelle de $28{ }^{\circ} \mathrm{C}$. L'humidité relative moyenne est inférieure à $50 \%$ durant les mois secs de l'année et l'évapotranspiration est accrue pendant 9 mois de l'année. La végétation naturelle est une savane arborée à combrétacées et à légumineuses avec une cinquantaine d'espèces d'arbres et d'arbustes dont Vitellaria paradoxa Gaertn, Khaya senegalensis Desr, Parkia biglobosa Jacq, Tamarindus indica L, Kigelia africana Lam, (Lodoum, 2000) auxquelles vient s'ajouter Cassia siamea Lam. La strate herbeuse est haute et dominée par diverses espèces d'Andropogon spp, Pennisetum Trin, 


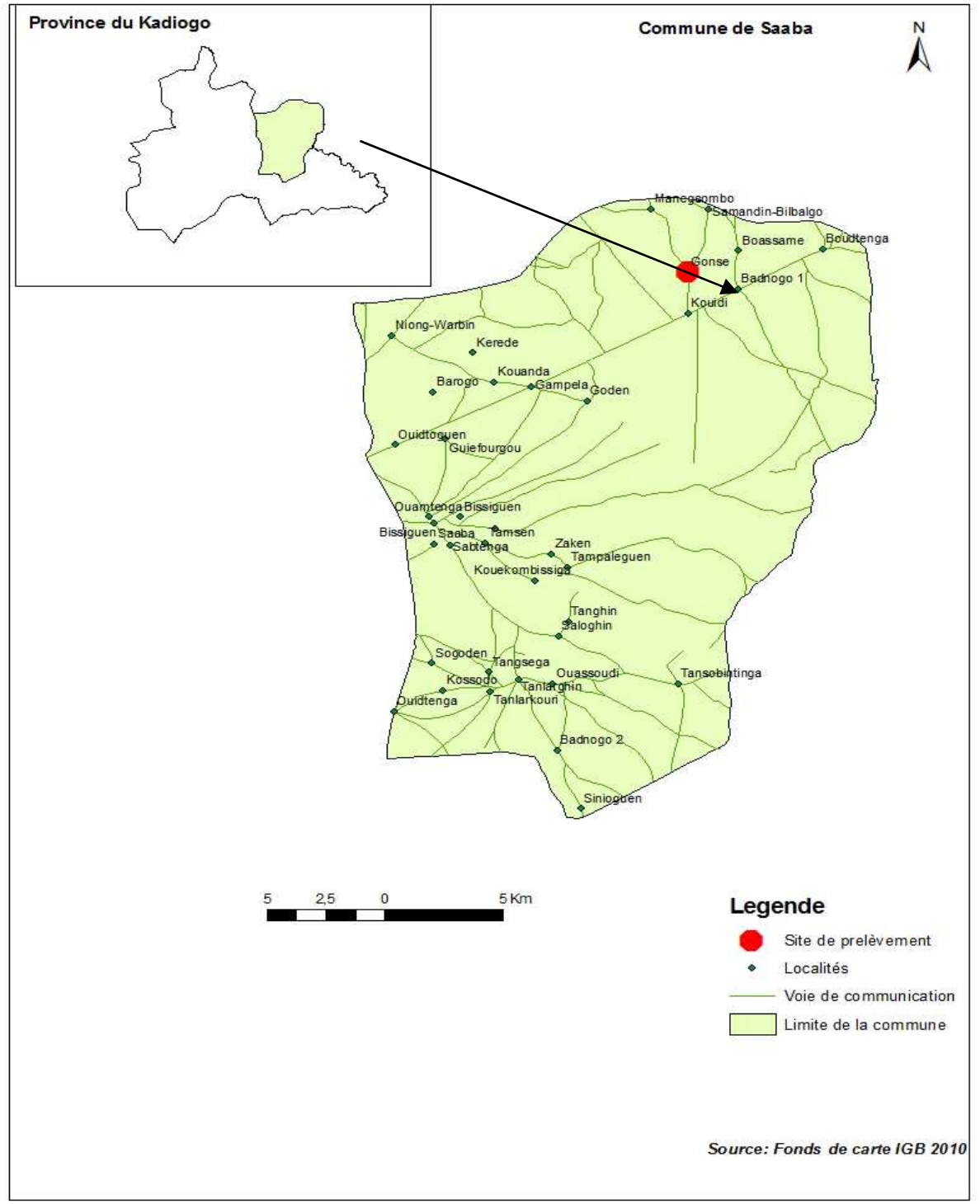

Figure 1: Situation géographique du site de prélèvement.

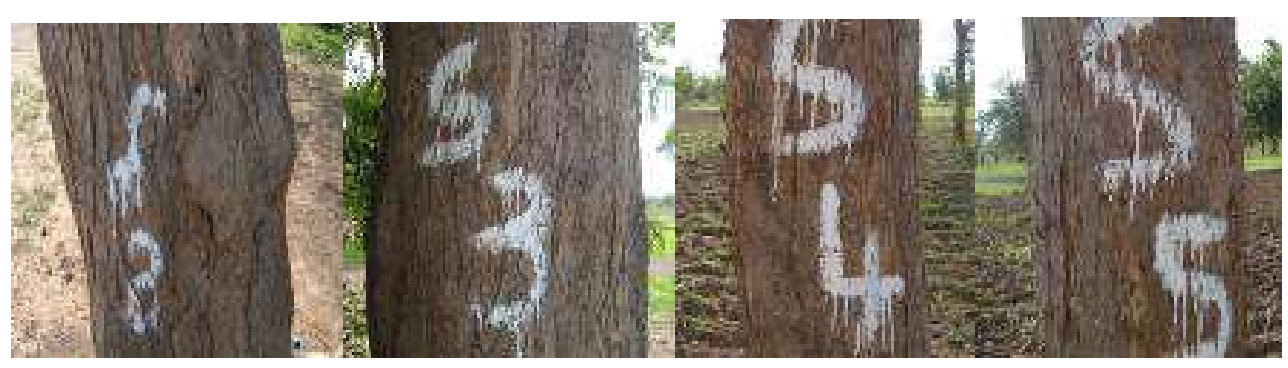

Photo 1 : Sujets de Faidherbia albida marqués. 
Eragrostis tremula Hochst et Stylosantes eracta Beauv.

\section{Méthodes}

Le matériel végétal qui a servi pour l'étude est constitué d'échantillons de jeunes rameaux, d'écorces et de feuilles de Faidherbia albida.

Les prélèvements ont été effectués sur des sujets retenus sur la base du critère d'âge. Ainsi, des approximations d'âge ont été faites avec l'appui de la population locale et cinq (5) pieds saints ont été ciblés suivant la répartition ci-après:

- deux (2) sujets vieux: plus de 30 ans;

- un sujet moyennement âgé: 15-25 ans;

- deux (2) sujets relativement jeunes: inférieurs à 15 ans.

Après l'identification, les sujets ont été marqués à la peinture blanche (Photo 1) et les points géographiques correspondant à chaque sujet ont été relevés avec un GPS de marque Garmin.

Durant la saison pluvieuse de l'année 2011 qui a duré de juin à octobre, une série d'observations qualitatives portant sur l'évolution de la défeuillaison, la réapparition des feuilles et des organes floraux a été faite sur les sujets marqués.

Les différents échantillons ont été prélevés sur des sujets sains durant la période de juin à septembre 2011 avec une fréquence de prélèvements de 15 jours. Les prélèvements sont effectués tôt le matin avant 8 heures pour réduire l'influence des rayons solaires sur le métabolisme de la plante. Cinq (5) séries de prélèvements ont été réalisées. Au total, 75 échantillons ont été prélevés à raison de 15 échantillons par organe. Les écorces et les rameaux sont prélevés à l'aide de machettes et de pioches. Le prélèvement des feuilles a nécessité l'utilisation d'une gaule pour permettre d'atteindre celles situées en hauteur surtout pour les sujets âgés. La partie ouest des arbres est moins exposée aux intempéries de la saison pluvieuse qui pourraient souiller les blessures. De ce fait, pour permettre une cicatrisation rapide des blessures, tous les prélèvements ont été faits dans cette partie des pieds choisis. Les échantillons enveloppés dans des sachets plastiques ont été ensuite conservés au congélateur à $-20^{\circ} \mathrm{C}$.

Les techniques d'extraction et de dosage ont été réalisées selon la méthode adaptée de Leroux (1984) et de Maldiney (1988). Après le dosage d'échantillons de $20 \mathrm{~g}$ de matières végétales fraîches, la lecture en 2 répétitions est faite par spectrophotométrie étalonné à $650 \mathrm{~nm}$. La détermination des différentes concentrations en hormones est faite à partir d'une courbe d'étalonnage d'équation $\mathrm{y}=\mathrm{ax}+\mathrm{b}$.

\section{Analyse des données}

Les données ont fait l'objet d'une analyse de variance avec le logiciel XL STAT 7.5.2 version 2004. Les moyennes ont été comparées en utilisant le test de NEWMAN et KEULS au seuil de $5 \%$.

\section{RESULTATS}

\section{Observations phénologiques}

Les observations faites de juin à octobre 2011 ont permis de noter une perte progressive des feuilles durant toute la période. Cette défeuillaison était plus visible au niveau des pieds relativement jeunes. En effet, ces pieds ont perdu presque toutes leurs feuilles à la fin du mois de juillet. Par contre, les vieux pieds et ceux qui ont subi un émondage au cours des saisons précédentes ont conservé des feuilles sur quelques branches durant toute la saison. A la fin du mois de septembre, les feuilles ont commencé à apparaître sur tous les pieds. En fin octobre, tous les pieds suivis étaient presque entièrement couverts de feuilles. Il n'y pas eu de floraison durant la période de l'étude.

\section{Teneurs en phytohormones des différents organes \\ Les teneurs en AIA3}

L'évaluation des teneurs en auxine principalement l'AIA3 dans les organes 
comme les jeunes rameaux, l'écorce et les feuilles de Faidherbia albida au cours de la saison pluvieuse (Figure 2), révèle de très faibles teneurs dans ces 3 organes $(<0,5$ $\mathrm{mg} / \mathrm{kg}$ de matière végétale fraîche) en juin, début effectif de la saison pluvieuse. Ces teneurs deviennent relativement importantes au niveau de l'écorce, environ $1,5 \mathrm{mg} / \mathrm{kg}$ de matière végétale fraîche après la deuxième semaine du mois d'août. Par contre, elles restent faibles au niveau des rameaux à cette même période. Cette faible teneur serait liée à la nature de l'organe prélevé et au taux élevé de gibbérelline (Polit et al., 2003).

Les teneurs en GA3

La concentration moyenne en gibbérelline GA3 dans l'écorce de Faidherbia albida a varié de 1,6 à $2,2 \mathrm{mg} / \mathrm{kg}$ de matière végétale fraîche de la dernière semaine du mois de juin à la première semaine du mois d'août (Figure 3). Dans les jeunes rameaux, la teneur en GA3 est inférieure à $2 \mathrm{mg} / \mathrm{kg}$ en fin juin et des teneurs plus faibles durant le mois de juillet. Dans les feuilles par contre, la teneur en GA est relativement plus importante que dans l'écorce et dans les jeunes rameaux. En effet, les teneurs en GA3 dans les feuilles ont varié entre $1,87 \mathrm{mg} / \mathrm{kg}$ et $2,35 \mathrm{mg} / \mathrm{kg}$ de fin juin à début août pour atteindre une valeur de $3 \mathrm{mg} / \mathrm{kg}$. Au seuil de signification de $5 \%$, l'analyse de variance révèle une différence significative entre les feuilles et les jeunes rameaux d'une part et entre les feuilles et l'écorce d'autre part (Tableau 1) avec une teneur relativement élevée dans les feuilles. Selon Thomas et al. (2005), la teneur en gibbérelline élevée dans les feuilles est imputable à la nature de l'organe.

\section{Les teneurs en kinétine}

Quant à la teneur en kinétine (cytokinine), elle est relativement élevée, plus de $2 \mathrm{mg} / \mathrm{kg}$ de matière végétale fraîche dans tous les organes prélevés en début de saison de pluies (fin juin) et dans la troisième semaine du mois d'août avec une teneur plus importante au niveau des feuilles (Figure 4). Mais, durant le mois de juillet et en début août, il apparaît des teneurs en kinétine faibles dans les trois organes étudiés. Selon le test de Newman-Keuls au seuil de 5\%, les différents organes n'ont pas d'effet significatif sur la teneur en kinétine de Faidherbia albida (Tableau 1).

\section{Teneurs comparatives en phytohormones dans l'écorce}

Les teneurs moyennes en phytohormones révèlent que la teneur en auxine (AIA3) est largement inférieure aux teneurs en gibérrelline (GA3) et en kinétine (cytokinine) dans l'écorce du tronc de Faidherbia albida tout comme dans ses feuilles et ses jeunes rameaux de fin juin à miaoût (Figure 5). L'évolution des teneurs en kinétine et en AIA3 sont relativement faibles de fin juin jusqu'en fin juillet. Mais, à partir du mois d'août, ces deux hormones ont connu une hausse remarquable (Figure 5). La teneur en gibbérelline GA3 a évolué en hausse durant le mois de juillet avant de baisser de façon progressive au mois d'août. Des trois hormones, la teneur en AIA3 reste faible comparativement à la kinétine et à la GA3, bien qu'elle ait connu une augmentation en août.

\section{Teneurs comparatives en phytohormones dans les rameaux \\ Dans les rameaux, les teneurs en} gibbérelline GA3 présente une hausse à partir de juillet mais a baissé à partir de début août. Celle en kinétine présente de grande fluctuation (Figure 6). La teneur en auxine a connu une hausse à partir de juillet jusqu'en août.

\section{Teneurs comparatives en phytohormones dans les feuilles \\ L'évolution des teneurs en} phytohormones dans les feuilles révèle une constance de 1'AIA3 de juin en début août puis une augmentation sensible à partir de la deuxième quinzaine du mois d'août. Quant à la gibbérelline GA3 et à la kinétine, leurs teneurs a commencé à augmenter en fin juillet 
après une évolution disparate en début de saison (Figure 7).

L'analyse en composantes principales (ACP) indique des différences significatives entre les différents organes avec un coefficient de variation de 5,5. Il apparaît une influence de la synthèse de l'auxine AIA3 sur celle de la gibbérelline GA3. Il est à noter que la teneur en auxine AIA3 ou de la gibbérelline GA3 n'influence pas celle de la kinétine. Les teneurs en kinétine et en auxine AIA3 sont statistiquement identiques (Figure 8).

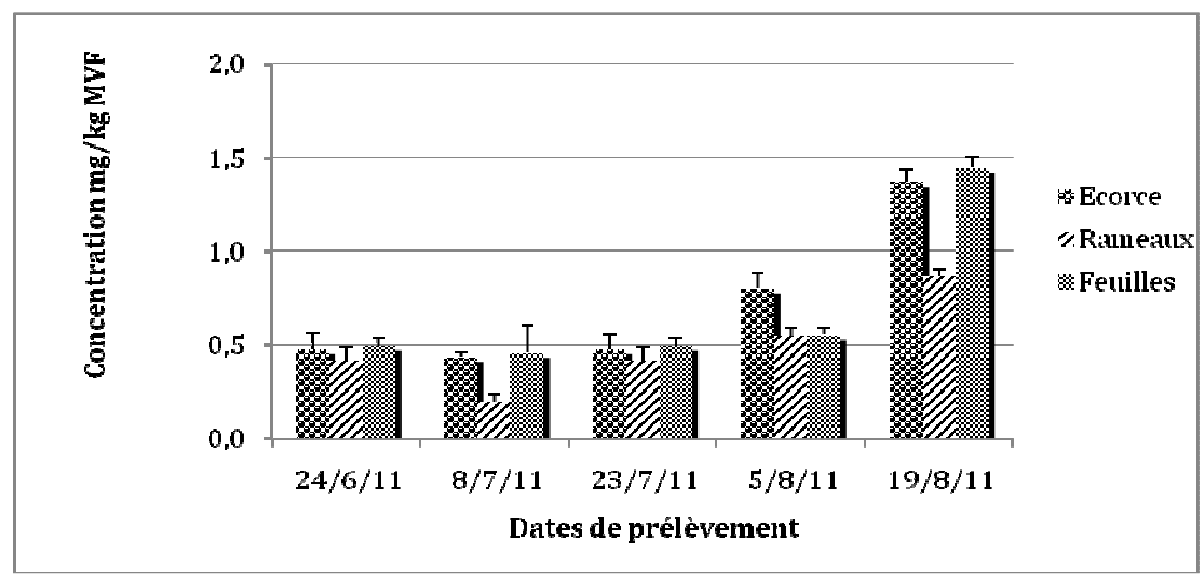

Figure 2: Teneur en auxine AIA3 des différents organes au cours de la saison pluvieuse. MVF : matière végétale fraîche.

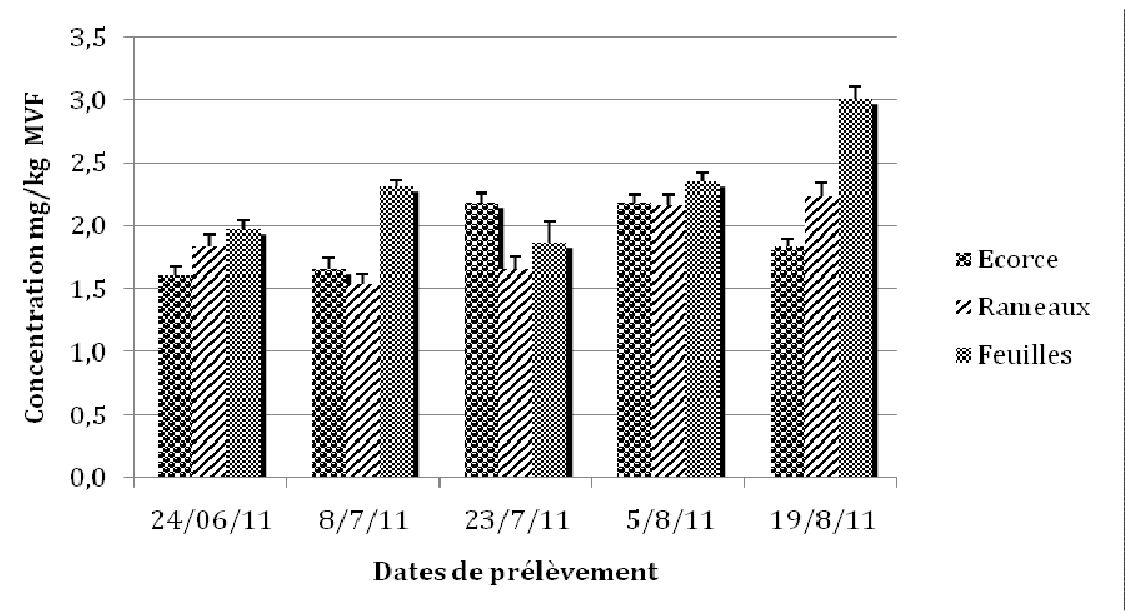

Figure 3 : Teneur en gibbérelline GA3 dans les différents organes. 


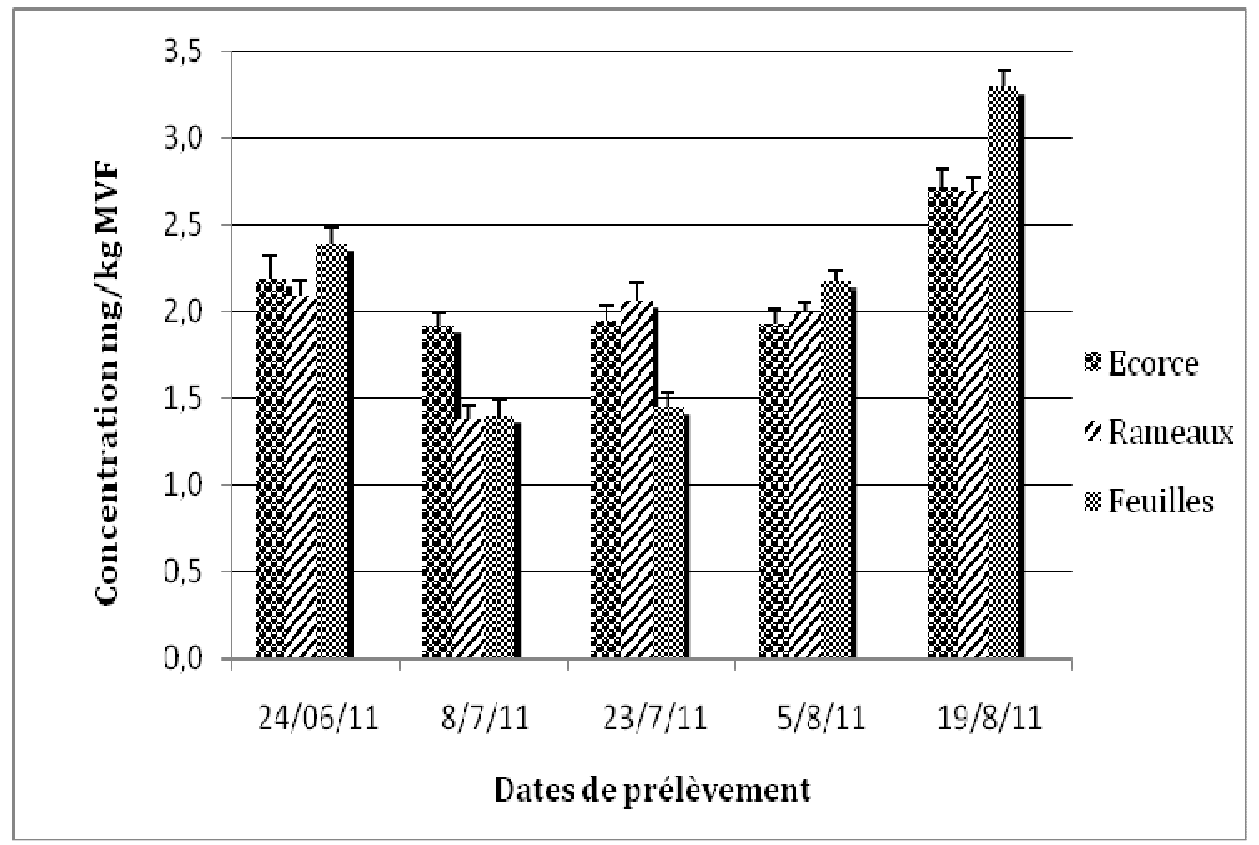

Figure 4: Teneur en kinetine dans les différents organes.

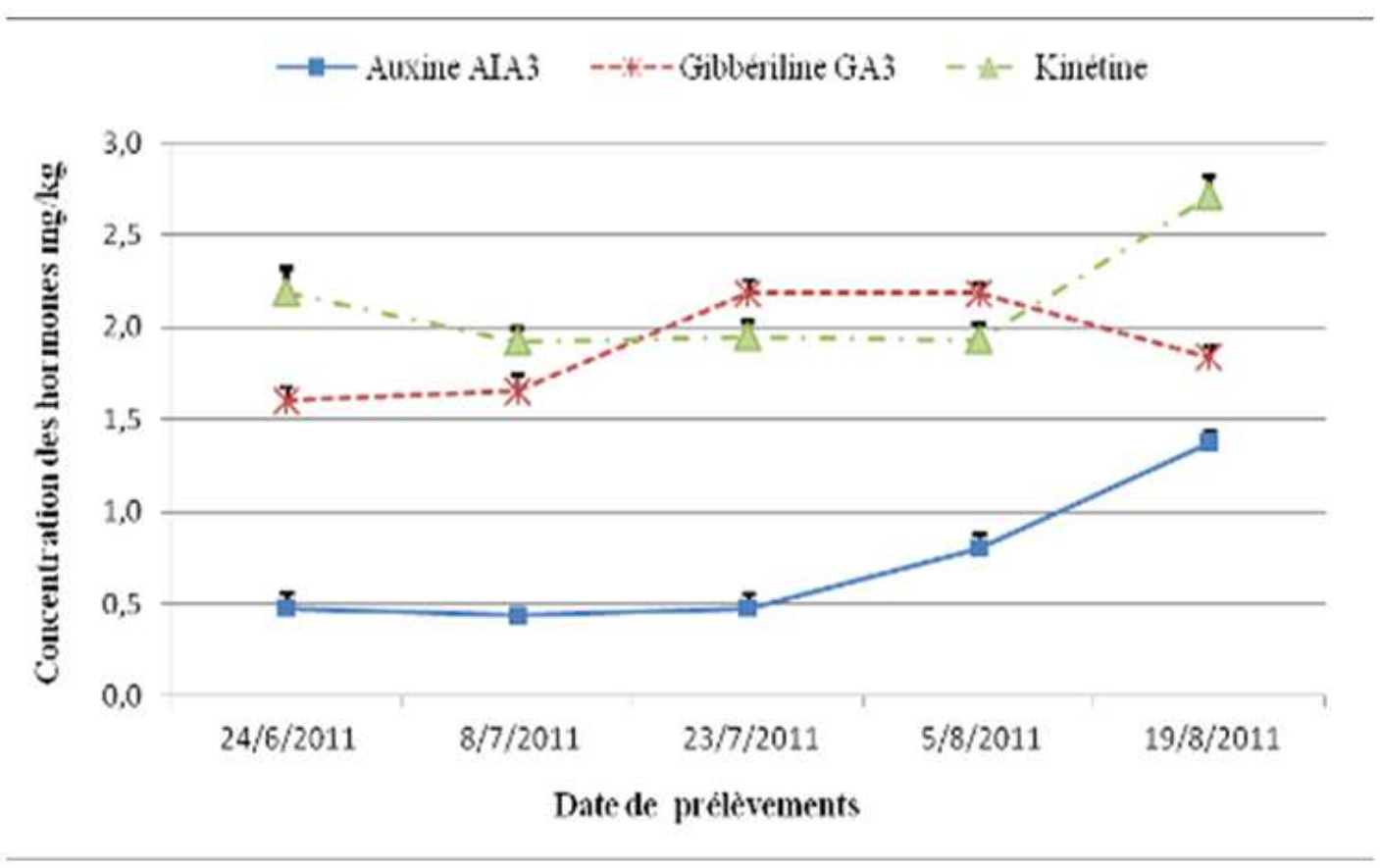

Figure 5:Evolution des teneurs en hormones au niveau de l'écorce du tronc. 


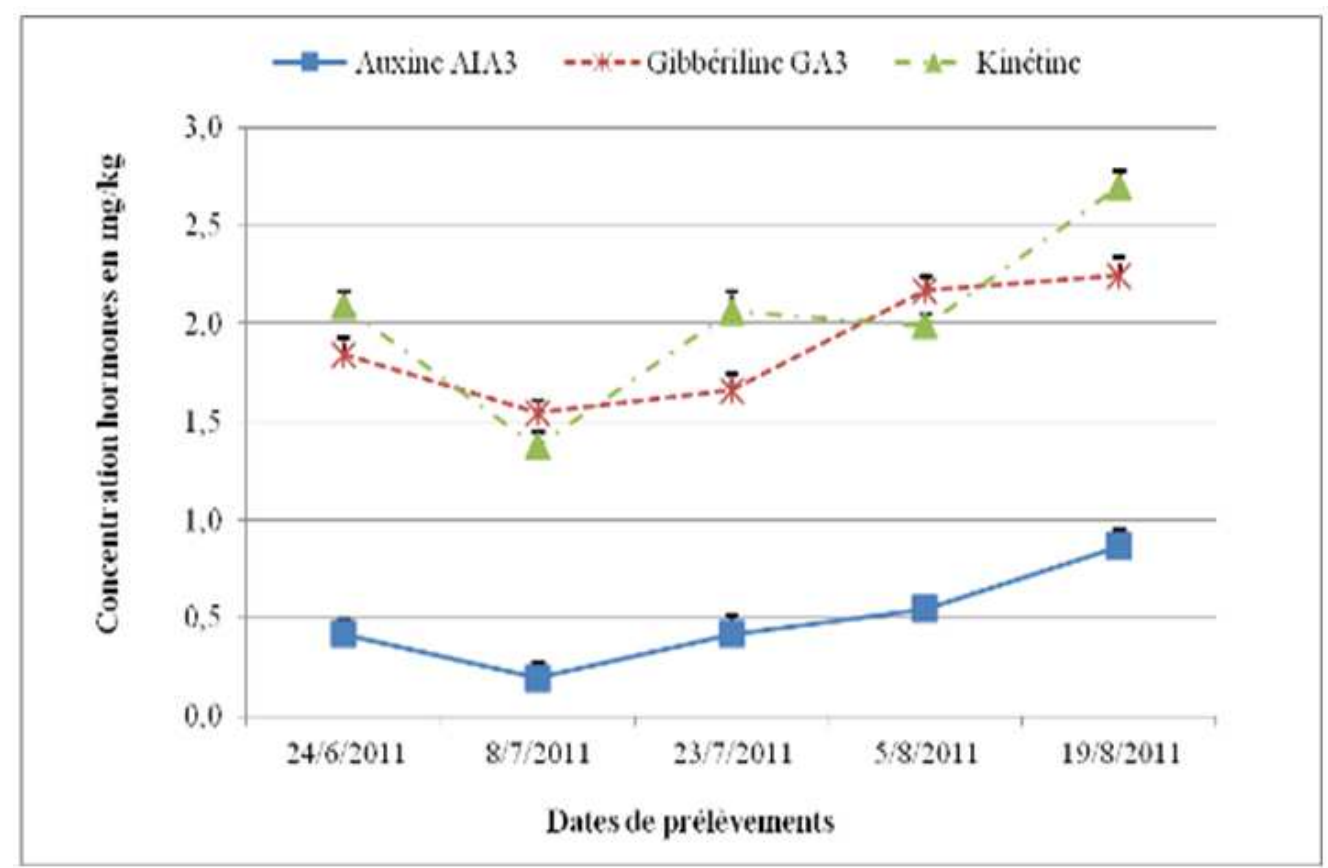

Figure 6: Evolution des teneurs en hormones au niveau des rameaux.

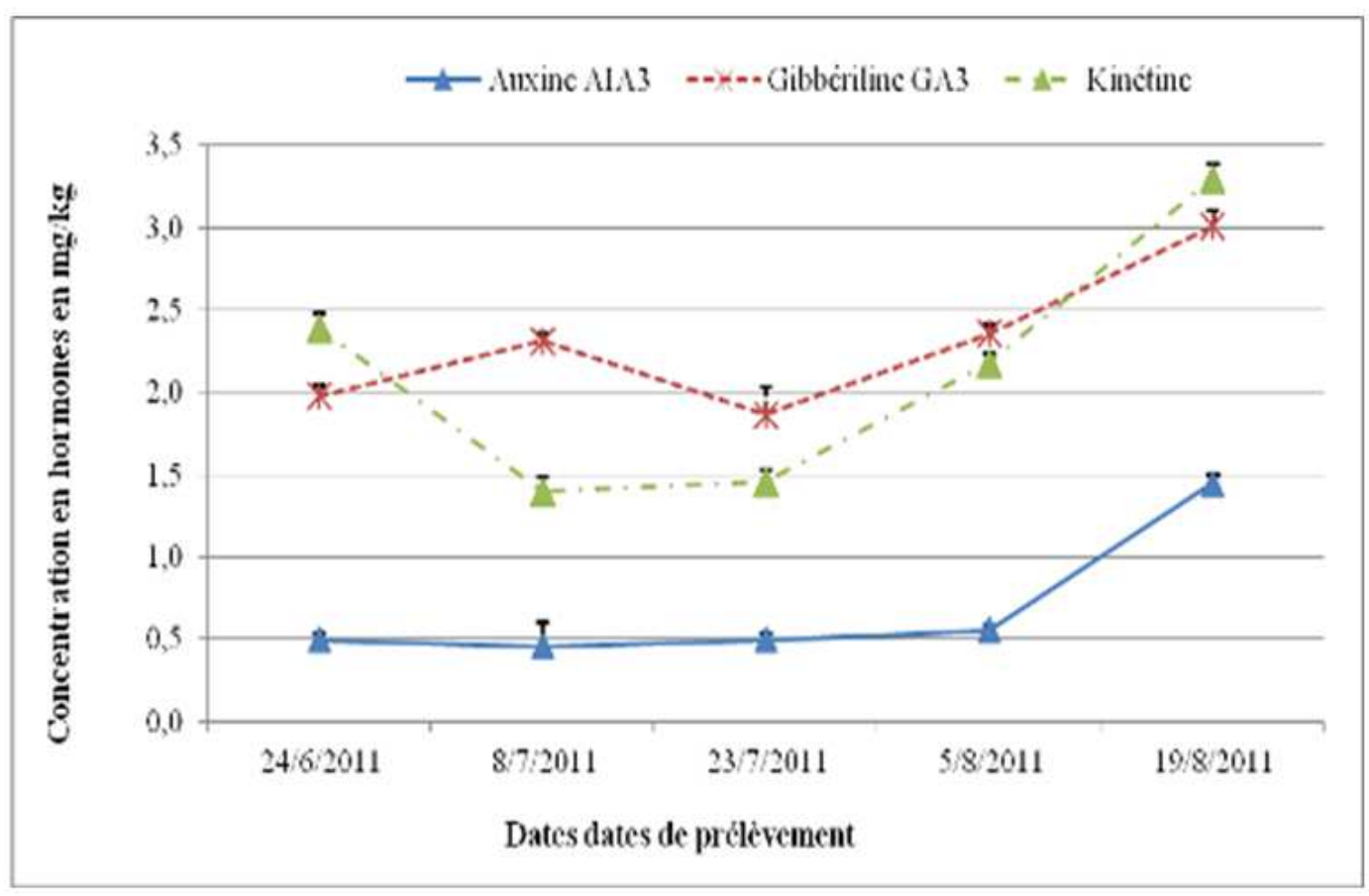

Figure 7 : Evolution des teneurs en hormones au niveau des feuilles. 


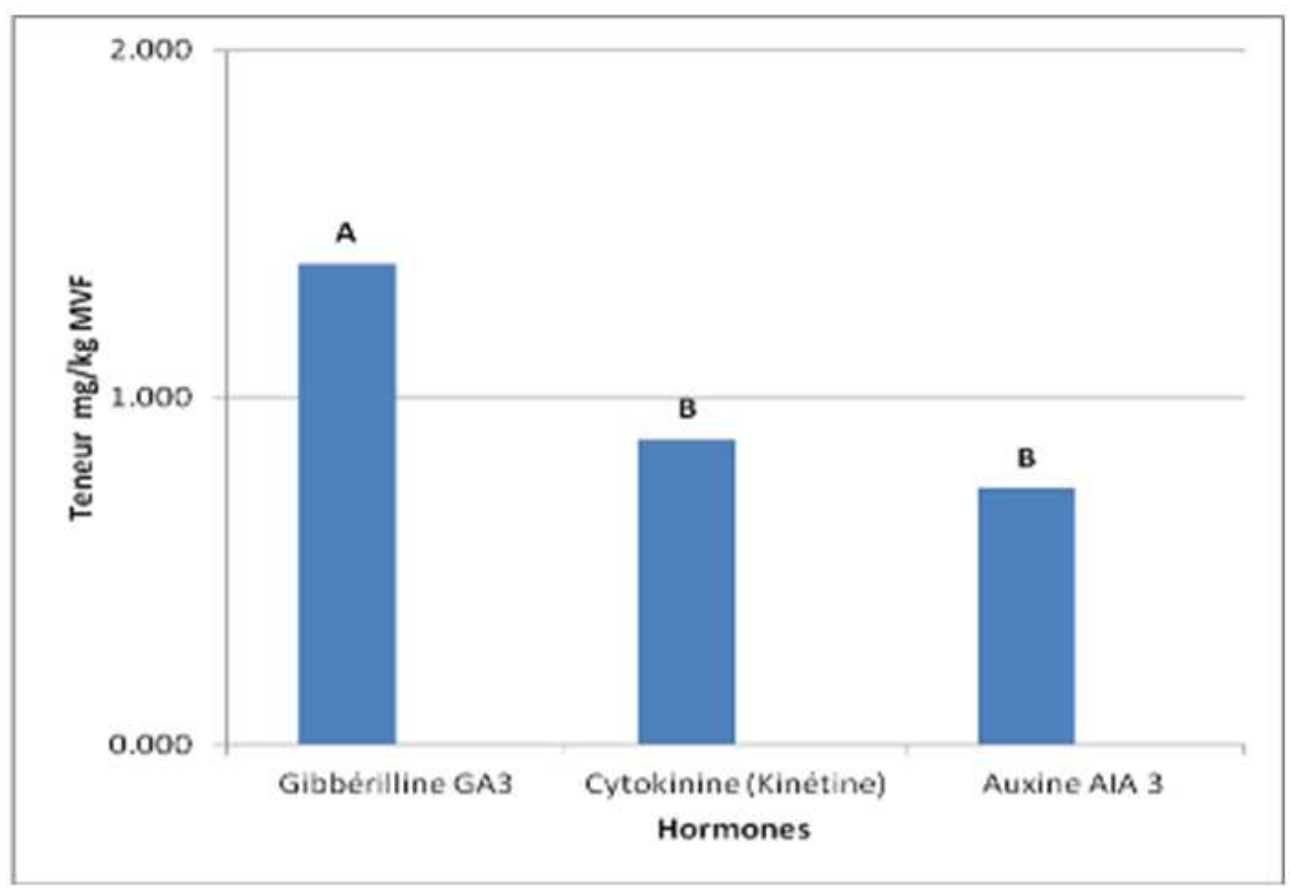

Figure 8: Variabilité entre les différentes hormones.

Tableau 1: Teneur en phytohormones dans les différents organes.

\begin{tabular}{lccc}
\hline \multirow{2}{*}{ Organes } & \multicolumn{3}{c}{ Hormones végétales } \\
\cline { 2 - 4 } & Auxine (AIA3) & $\begin{array}{c}\text { Gibbérelline } \\
(\mathbf{G A 3})\end{array}$ & Kinétine \\
\hline Feuilles & $0,69^{\mathrm{a}}$ & $2,30^{\mathrm{a}}$ & $2,11^{\mathrm{a}}$ \\
Jeunes rameaux & $0,49^{\mathrm{a}}$ & $1,94^{\mathrm{b}}$ & $2,15^{\mathrm{a}}$ \\
Ecorce & $0,71^{\mathrm{a}}$ & $1,89^{\mathrm{b}}$ & $2,09^{\mathrm{a}}$ \\
\hline
\end{tabular}

\section{DISCUSSION}

Les résultats ont montré une teneur en auxine AIA3 relativement faible (0,5-1,4 $\mathrm{mg} / \mathrm{kg}$ ) dans tous les organes aériens. Ces teneurs avoisinent celles obtenues par Vassal (2001) sur Acacia radiana $(1,8 \mathrm{mg} / \mathrm{kg})$. Cependant, Alexandre et al. (2005) obtinrent des teneurs en auxine plus élevées dans les feuilles d'Acacia senegal (4 mg/kg).

La faible teneur en auxine AIA3 dans les organes de Faidherbia albida serait liée au taux élevé de gibbérelline dans ces organes. Policit et al. (2003) ont attribué le faible taux d'auxine dans les organes végétaux à la teneur élevée en gibbérelline ou en kinétine d'une part et d'autre part à la nature des organes prélevés. Selon ce dernier auteur, l'auxine serait en faible concentration car elle constituerait essentiellement un élément précurseur de la synthèse des autres hormones végétales. En outre, l'auxine est prédominante dans les apex des tiges, dans les méristèmes et dans les jeunes feuilles des bourgeons terminaux. Besse (2000) soulignait aussi que les teneurs en auxine variaient selon les organes et les périodes de développement de la plante. En effet, l'AIA-3 active la meresis au niveau cambial et lorsque sa concentration 
est élevée du côté distal du pétiole, il inhibe l'abscission. Sa forte teneur dans les feuilles, vers la fin de la saison pluvieuse aurait pour conséquence le ralentissement de la chute des feuilles qui ont persisté.

Les concentrations en gibbérelline GA3 obtenues sont plus élevées $(1,5-3 \mathrm{mg} / \mathrm{kg})$ que celles en auxine $(0,3$ à $1,45 \mathrm{mg} / \mathrm{kg})$. Une différence significative a été notée entre les teneurs en gibbérelline des feuilles et celle des autres organes notamment les écorces et les rameaux. Ces résultats se rapprochent de ceux obtenus par Vassal (2001) sur Acacia radiana $(1,4 \mathrm{mg} / \mathrm{kg})$ mais apparaît plus faibles par rapport à ceux trouvés par Alexandre et al. (2005) sur l'espèce Acacia senegal (3,5 $\mathrm{mg} / \mathrm{kg}$ ). Cette différence de résultats serait liée à la nature de l'organe analysé. En effet, la gibbérelline est synthétisée dans plusieurs régions de la plante et particulièrement au niveau des zones actives de division cellulaire (Besse, 2000). Selon Heller (2005), la gibbérelline induirait la croissance des feuilles.

La teneur en kinétine a montré une fluctuation suivant les organes $(1,5-3,5 \mathrm{mg} / \mathrm{kg}$ de matière végétale fraîche). Cependant, aucune différence significative n'est constatée. Les teneurs en kinétine sont faibles par rapport à celles trouvées par Vassal (2001) sur Acacia radiana $(1,8 \mathrm{mg} / \mathrm{kg})$ et Alexandre et al. (2005) sur Acacia senegal $(4,2 \mathrm{mg} / \mathrm{kg})$. Cette faible teneur peut être liée à l'espèce mais aussi au fait que la kinétine est beaucoup plus synthétisée au niveau de l'apex racinaire (Besse, 2000).

Quant à l'évolution des différentes hormones au niveau des organes, elle présente de grandes disparités suivant les organes. Les résultats montrent l'existence de corrélations de 0, 6 entre l'auxine AIA3 et la gibbérelline GA3. Des travaux antérieurs ont montré une parfaite corrélation entre les principales hormones (Heller, 2005).

En effet, les teneurs en AIA3, kinétine et gibbérilline obtenues sont également observables chez d'autres espèces telles que Acacia senegal et Acacia radiana de la même famille que Faidherbia albida (Vassal, 2001).
D'après les travaux de Heller (2005), l'apparition des bourgeons serait liée à un rapport kinétine sur AIA-3 égal = 100environ. Cela pourrait expliquer le redémarrage de la feuillaison à la fin de la saison pluvieuse mais il n'empêche que la défeuillaison de Faidherbia albida serait liée aux conditions biophysiques du milieu. En effet, Traoré (2006) a estimé que la périodicité, l'abondance et la durée des pluies pouvaient déterminer l'apparition des feuilles de Faidherbia albida. Nos résultats pourraient être expliqués par le facteur "pluies" qui a connu une évolution difficile et un arrêt précoce en septembre 2011.

Une autre composante majeure influençant la dynamique de feuillaison est l'aspect génétique émis par le même auteur qui, après un suivi de l'espèce a estimé que l'inversion de la phénologie est imputable à des facteurs génétiques. Par ailleurs, Besse (2000) démontre que la teneur en hormones de la plante est fortement influencée par la teneur en eau et en éléments nutritifs du sol.

\section{Conclusion}

L'importance de Faidherbia albida dans la vie socio-économique a suscité cette étude par l'utilisation de méthodes biotechnologiques. L'étude a été conduite en vue de contribuer à une meilleure connaissance de la physiologie de l'espèce Faidherbia albida. Ainsi, les résultats obtenus ont montré une fluctuation de la synthèse d'hormones dans les différents organes aériens. L'étude a révélé des teneurs assez variables en phytohormones durant la saison des pluies. Elle a en outre montré une évolution disparate de ces hormones au cours de la saison et en fonction des organes végétatifs. Les résultats obtenus laissent présager qu'il existerait une influence quelconque sur le comportement de la plante. En effet, les résultats obtenus laissent supposer à priori une certaine dynamique corrélative entre les différentes hormones sur le comportement végétatif de Faidherbia albida. Ces résultats ont permis d'avoir de nouvelles informations sur la physiologie de 
l'espèce. Cette dynamique corrélative pourrait être complétée par des prélèvements à différentes dates de la saison sèche pendant laquelle il y a abondance des feuilles.

\section{REFERENCES}

Alexandre I, Dominique F, Hubert G. 2005. Effect of kinetin on protein, nucleic acid metabolism in Acacia Senegal leaves during senescence. Plant Physiology, 37: 595-602.

Bationo BA, Ouédraogo JS, Alexandre DY, Guinko S. 2001. Statut hydrique de quatre espèces ligneuses soudaniennes dans la forêt de Nazinon, Burkina Faso. Science et changements planétaires/Sécheresse. 87-94.

Besse L. 2000. Recherche du déterminisme hormonal de l'anomalie de la morphogenèse florale liée à l'embryogenèse somatique chez le palmier à huile (Elaeis guineensis Jacq.). Thèse de Doctorat. Université Paris VI, p. 158 .

FAO. 2000. Annuaire FAO de la production collection FAO statistiques, p.163.

Gnankambary Z. 2007. Compost and fertilizer mineralization effects on soil and Harvest in arkland Agroforestry Systems in the South-Sudanese Zone of Burkina Faso. $\mathrm{PhD}$, Swedish University of Agricultural Sciences, Ume, p.47.

Harivel A, Bellefontaine R, Boly O. 2006. Aptitude à la multiplication végétative de huit espèces forestières d'intérêt au Burkina Faso. Bois et Forêts des Tropiques, 288: 39-50

Heller R. 2005. Physiologie végétale -2Développement ( 3 ème édn). Masson: Paris; 215.

Khao RM,Yacouba B, Yaye M, Katkore B, Moussa A, Iktam A, Mayaki A. 2001. Separating the effects of trees on crops: the case of Faidherbia albida and millet in Niger. Agroforestry Systems, 52: 219238.
Leroux B. 1984. Recherche méthodologique sur le dosage immunoenzymatique de trois substances de croissance végétale: l'auxine, l'acide abscissique et la zéatineriboside. Thèse de Docteur Ingénieur, INA-Paris Grignon, p.110.

Lodoum T. 2000. Etude de quelques espèces nectarifères et pollinifères de la formation végétale de Gampèla (Burkina Faso) Mémoire DEA, Université de Ouagadougou, p.71.

Maldiney R. 1988. Nouvelle approche méthodologique de dosage et de la localisation de phytohormones par des techniques immunologiques. Application à la tomate, Lycopersicon esculentum Mill, cv. Craigella et à un mutant, lateral suppressor. Thèse de Doctorat d'Etat, Université PM. Curie, Paris, p.191.

Polit JT, Maszewski J, Kazmierczak A. 2003. Effect of BAP and IAA on the expression of G1 and G2 control points and G1-S and $\mathrm{G} 2-\mathrm{M}$ transitions in root meristem cells of Vicia faba. Cell Biology International, 7: 559-566.

Ouédraogo S. 2004. Observation sur la phénologie d'Acacia albida à Kokologho (Burkina Faso), physiologie des arbres et arbustes en zones semi arides. Mémoire d'étude, Université de Ouagadougou, p.53.

Thomas SG, Rieu I, Steber CM. 2005.Gibberellin metabolism and signaling. Vitam Horm, 72: 289-338.

Traoré H. 2006. Contribution à l'étude de la diversité écologique Acacia albida à l'ouest du Burkina Faso. Mémoire de DEA, Université Polytechnique de Bobo Dioulasso, p.49.

Vassal J, 2001. Etat de connaissances sur l'induction de gommose chez Acacia radiana. Physiologie des arbres et arbustes en zones arides et semi-arides: séminaire Paris-Nancy, 20 mars-6 avril 2000. Libbey, Paris, France, 271-276. 